\title{
Pre-Post Effects of the
}

\section{Psychoeducational, Autism-Specific Parent Training FAUT-E}

\author{
Juliane E. Medda ${ }^{1, a}$, Janina Kitzerow ${ }^{1, a}$, Sabine Schlitt ${ }^{1}$, Kerstin Berndt ${ }^{1}$, \\ Christina Schwenck ${ }^{1,2}$, Lorenz Uhlmann³ ${ }^{3}$, and Christine M. Freitag ${ }^{1}$
}

\author{
Department of Child and Adolescent Psychiatry, Psychosomatics and Psychotherapy, Autism Research and Intervention Centre \\ of Excellence, University Hospital Frankfurt, University of Frankfurt/Main \\ Department of Clinical Child and Adolescent Psychology, University of Giessen \\ Institute of Medical Biometry and Informatics, University of Heidelberg \\ a Shared first authors
}

\begin{abstract}
Objective: Psychoeducational parent training is an economic way to provide care for parents of children newly diagnosed with an autism spectrum disorder (ASD). This study explores pre-post effect sizes of the manualized autism-specific parent training FAUT-E (Frankfurter Autismus-Elterntraining). Method: Two behaviorally trained therapists worked with 6-10 parents in eight group sessions. Twenty-four parents of 24 children with ASD participated in the study. Outcomes were child- and parent-related measures obtained at T0 (first measurement), T1 (second measurement), T2 (postintervention), and T3 (3 months after intervention). Results: Children showed improved behavior in the parentrated Aberrant Behavior Checklist (ABC) total score after therapy $(p=.001$; ES T1T2 $=.73)$ and at T3 $(p=.018$; ES T1-T3 $=-.51)$, and a lower intensity of parent-rated problem behavior at T3 $(p=.031$; ES T1-T3 $=-.46)$. Parental measures did not change. Conclusions: This study found medium pre-post effects on the child's behavior by FAUT-E between T1 and T2/T3; these were not observed between the measurements T0-T1. FAUT-E was easy to implement and did not increase parental stress. This is in line with results of studies on other training programs to teach parents to use effective behavioral strategies with ASD.
\end{abstract}

Keywords: autism spectrum disorder, FAUT-E, parent training, psychoeducation, short-term intervention

Prä-post-Effekte des Autismus-spezifischen psychoedukativen Elterntrainings FAUT-E

Zusammenfassung: Fragestellung: Psychoedukative Elterntrainings sind ein kostengünstiges Behandlungsangebot für Eltern von Kindern mit einer neu diagnostizierten Autismus-Spektrum-Störung. Das Ziel dieser Studie war die Untersuchung von Prä-post-Effektgrößen des manualisierten Frankfurter Autismus-Elterntrainings (FAUT-E). Methodik: Zwei Therapeuten leiteten acht Gruppensitzungen mit 6-10 Eltern. Vierundzwanzig Eltern von 24 Kindern mit ASD wurden eingeschlossen. Kind- und elternbezogene Daten wurden bei T0 (erste Messung), T1 (zweite Messung), T2 (nach Intervention) und T3 (3 Monate nach Intervention) erhoben. Ergebnisse: Die Ergebnisse des Gesamtwertes des Elternfragebogens Aberrant Behavior Checklist (ABC) verbesserten sich nach Therapie ( $p=.001$; ES T1-T2 $=-.73$ ) und zu T3 ( $p=.018$; ES T1-T3 = -.51). Zusätzlich berichteten die Eltern zum Zeitpunkt T3 über ein reduziertes kindliches Problemverhalten ( $p=.031 ;$ ES T1-T3 = -.46). Die ElternmaBe zeigten keine Veränderung. Diskussion: Das autismusspezifische psychoedukative Elterntraining FAUT-E war gut im klinischen Alltag umsetzbar. Die Eltern berichteten mittlere Prä-post-Effekte auf das kindliche Verhalten im Elternurteil, die sich von den Änderungen der ersten Messungen (TO-T1) unterschieden. Der elterliche Stress stieg durch die Intervention nicht an. Die Ergebnisse sind vergleichbar mit anderen verhaltenstherapeutisch basierten Elterntrainings, die Eltern von ASD-Patienten effektive Erziehungsstrategien vermitteln.

Schlüsselwörter: Autismus-Spektrum-Störung, FAUT-E, Elterntraining, Psychoedukation, Kurzintervention

Autism spectrum disorders (ASD) are pervasive developmental disorders characterized by impaired social communication and stereotyped, repetitive behavior (American Psychiatric Association, 2013). In addition to core ASD symptoms, many behavioral problems accompany the di- agnosis of ASD which can severely impact the child's and the family's well-being (Fairthorne et al., 2015). In the British NICE guidelines, the evaluation of group-based parent trainings with the aim of reducing early emerging challenging behavior was defined as an important area of fu- 
ture research (Kendall et al., 2013). Similarly, another paper advocated the development and evaluation of brief behavioral parenting interventions that can be rapidly implemented in easily accessible health care settings (McMillin et al., 2015). Especially hyperactive, anxious, oppositional, and aggressive behaviors as well as the related comorbid psychiatric diagnoses are frequently observed in children with ASD (Simonoff et al., 2012).

To meet this need for immediate, cost-effective, and targeted interventions, several behaviorally based short-term group trainings for parents have been developed aiming at teaching parents about ASD and improving parenting strategies to effectively support the child and to handle emotional and behavioral problems of the child. Among these are the Stepping Stones Triple P program for autism (Whittingham et al., 2009; Schrott et al., 2019; Sprenger et al., 2015) the parent education and behavior management intervention (PEBM; Tonge et al., 2006; Tonge et al., 2014), the Happy Parenting Program (HPP; Leung et al., 2016), COMPASS for Hope (C-Hope; Kuravackel et al., 2018), Autism Spectrum Conditions - Enhancing Nurture and Development (ASCEND; Pillay et al., 2011), CYGNET (Stuttard et al., 2016), and another study comparing parent training to parent education only (Bearss et al., 2015; Iadarola et al., 2018).

These programs were developed for parents of children with ASD at various ages, such as preschool (Bearss et al., 2015; Iadarola et al., 2018; Kuravackel et al., 2018; Tonge et al., 2006; Whittingham et al., 2009), school age (Kuravackel et al., 2018; Pillay et al., 2011; Sprenger et al., 2015; Stuttard et al., 2016; Whittingham et al., 2009), preschool and school age (Schrott et al., 2019), or adolescence (Pillay et al., 2011; Stuttard et al., 2016). These programs target children with a wide range of cognitive abilities and comorbid behavior problems. In addition, the numbers of group and individual sessions differ between programs, ranging from seven (Stuttard et al., 2016) to 20 sessions (Tonge et al., 2006) in as many weeks. Some programs are exclusively group-based and offered at an intervention center (Kuravackel et al., 2018; Pillay et al., 2011; Stuttard et al., 2016), some add individualized coaching sessions delivered by telephone or in person (Kuravackel et al., 2018; Schrott et al., 2019; Sprenger et al., 2015; Tonge et al., 2006; Whittingham et al., 2009), while the parent training described by Bearss et al. is delivered in group settings and individually to the families in their homes (Bearss et al., 2015; Iadarola et al., 2018).

The main goal of most parent training is the reduction of child behavior problems (Bearss et al., 2015; Kuravackel et al., 2018; Leung et al., 2016; Pillay et al., 2011; Stuttard et al., 2016; Sprenger et al., 2015; Whittingham et al., 2009). Reduction in parent-reported disruptive behavior directly after the intervention was achieved by ASCEND, HPP, Cygnet, C-Hope, Stepping Stones Triple P, and the parent training described by Bearss et al. (Bearss et al., 2015; Kuravackel et al., 2018; Leung et al., 2016; Pillay et al., 2011; Stuttard et al., 2016; Whittingham et al., 2009). This effect was maintained at follow-up for HPP, Cygnet, and Stepping Stones Triple P and the parent training described by Bearss et al. (Bearss et al., 2015; Leung et al., 2016; Stuttard et al., 2016; Whittingham et al., 2009).

Other studies aimed exclusively (Tonge et al., 2006; Schrott et al., 2019) or additionally (Iadarola et al., 2018; Kuravackel et al., 2018; Leung et al., 2016; Sprenger et al., 2015; Stuttard et al., 2016; Whittingham et al., 2009) at increasing parental competence and reducing parental stress. Reduction of parental stress was reported for HPP (Leung et al., 2016), C-Hope (Kuravackel et al., 2018), Stepping Stones Triple P program for autism (Schrott et al., 2019), and the parent training described by Iadarola et al. (2018). A reduction of dysfunctional discipline strategies was achieved by implementing HPP and Stepping Stones Triple P, and was maintained at follow-up (Leung et al., 2016; , Schrott et al., 2019; Sprenger et al., 2015; Whittingham et al., 2009). PEBM led to a long-term reduction of anxiety and insomnia in parents (Tonge et al., 2006).

To date, no short-term program has been conceptualized to provide training to parents of all children and adolescents with ASD, irrespective of the child's age, language, symptom severity, or cognitive level. Also, most of the above-mentioned programs are not available in German. Therefore, we developed a manualized training for parents of children with ASD (FAUT-E; Schlitt et al., 2014). FAUT-E is an immediate, brief intervention offered directly after the child's first diagnosis for parents of all children and adolescents with ASD. It serves two purposes: informing parents about ASD and training parents in effective parenting strategies to support their children regarding their development as well as their adaptive and functional behavior. The training is delivered in a group setting in 8 sessions à 120 minutes by two behaviorally trained therapists over 3 months. Six basic modules are provided for all parents, and nine flexible modules can be chosen based on the needs of the group of parents regarding specific ASDrelated developmental aspects. The main aim of FAUT-E is to support parents in establishing a positive and nurturing parent-child interaction, based on the needs of the parents, the entire family, and the affected child. To achieve this goal, behaviorally based parenting strategies are taught to cope with autism-specific symptoms, developmental issues, and comorbid behavior problems. Another focus is the teaching of acceptance and commitment strategies. To meet the specific needs of the parents of children at different developmental stages, parents of children with relatively homogeneous characteristics are grouped together, such as parents of children with intellectual disability, preschool aged children, or high-functioning school- 
aged children or adolescents to better provide the respective relevant additional modules for each group. The aim of this study was to establish pre-post and 3-month follow-up effect sizes (ES) of FAUT-E on the child's behavior outcomes, parental well-being and parental competences.

\section{Methods}

\section{Study Design}

The study design was approved by the local Ethical Committee of the Medical Faculty of the University of Frankfurt/Main. After their child had received an ASD diagnosis at the Department of Child and Adolescent Psychiatry, Psychosomatics, and Psychotherapy, University Hospital Frankfurt, parents were informed about the parent training. The parents gave written informed consent to participate in the study. An uncontrolled repeated measures design with four measurement timepoints was implemented. Questionnaires were given to the parents 2 months before the training (T0; T1-T0: $\mathrm{M}=2.04$ months; $S D=0.7$ ), before start of the training (T1), after the training (T2; T2-T1: $\mathrm{M}=3.3$ months; $S D=0.7$ ), and at 2-3 months follow-up after the last training session (T3; T3-T2: $\mathrm{M}=2.56$ month; $S D=0.9$ ). Parents were asked to send all questionnaires back within 2 weeks. When more than one parent attended the training, the parent who attended the training most frequently was asked to fill out the questionnaires.

\section{Participants}

Study participation was optional for all parents attending the training between August 2012 and August 2015. The evaluation was carried out for 7 groups with 4-8 partici- pants per group. $N=40$ parents took part in the training during this period, and $80 \%$ of parents attended at least 6 out of 8 sessions (75\%). $N=37$ parents gave consent to participate in the study before the training started. Parents who did not return the $\mathrm{T} 1$ and $\mathrm{T} 2$ questionnaires completely and on time or who missed more than three appointments were excluded from the study $(N=13 ; 35 \%)$. Data of $N=24$ parents were included in the analysis. There was no difference between parents included and excluded regarding the following child characteristics: $\operatorname{sex}\left(\chi^{2}=.581 ; p=\right.$ $.446)$, diagnosis $\left(\chi^{2}=.4 .32 ; p=.229\right)$, IQ $(t=1.67 ; p=.103)$, or age at time of diagnosis $(U=-1.66 ; \mathrm{p}=.102)$. Children of parents in the drop-out group were younger than children of included parents $(U=-2.07 ; p=.039)$. Parents characteristics did not differ regarding socioeconomic status $(U=$ $-1.23 ; p=.224)$ or migration background $\left(\chi^{2}=1.90 ; p=\right.$ .387). More information on both group characteristics are shown in Table 1.

\section{Sample Characteristics}

A total of 20 mothers and 4 fathers $(16.7 \%)$ of 20 boys and 4 girls (16.7\%) with an ASD diagnosis according to ICD-10 criteria were included. ASD was diagnosed by an experienced clinician (child psychiatrist or clinical child psychologist), based on information provided by the parents in the Autism Diagnostic Interview-Revised (ADI-R; Bölte et al., 2006; Lord et al., 1994) and direct observation by the Autism Diagnostic Observation Schedule (ADOS; Lord et al., 1999; Rühl et al., 2004). Eight children were diagnosed with autism (F84.0; 33.3\%), six with atypical autism (F84.1;25.0\%), and ten children fulfilled criteria for an Asperger syndrome (F84.5; 41.7\%). Twelve children (50\%) had comorbid diagnoses. Of these children, four were di-

Table 1. Baseline characteristics of included study participants and dropouts.

\begin{tabular}{|c|c|c|c|c|c|}
\hline & & $\begin{array}{l}\text { Included partici- } \\
\text { pants }(N=24)\end{array}$ & $\begin{array}{l}\text { Dropped-out } \\
\text { participants }(N=13)\end{array}$ & $\begin{array}{l}t \text {-value/ } \\
U \text {-value }\end{array}$ & $p$-value \\
\hline & & $M(S D)$ & $M(S D)$ & & \\
\hline \multirow[t]{5}{*}{ Child characteristics } & Age at start of intervention & $9.42(8.6)$ & $7.5(2.6)$ & -2.07 & .039 \\
\hline & Age of ASD diagnosis & $8.56(2.9)$ & $6.76(1.8)$ & -1.66 & .102 \\
\hline & $\begin{array}{l}\text { Time between diagnosis } \\
\text { and start of FAUT-E (months) }\end{array}$ & $10.33(16.9)$ & $5.6(7.2)$ & -.808 & .425 \\
\hline & IQ & $92.00(22.2)$ & $77.75(27.6)$ & 1.67 & .103 \\
\hline & SRS Total $t$-score & $81.22(9.5)$ & $80.90(9.2)$ & .089 & .930 \\
\hline \multirow[t]{3}{*}{ Parent characteristics } & Age at start of intervention & $42.46(7.5)$ & $40.92(9.5)$ & .135 & .894 \\
\hline & Age at time of child's birth & $33.29(7.6)$ & $33.67(8.5)$ & -.534 & .597 \\
\hline & Socioeconomic status (HISEI) & $66.14(18.33)$ & $57.71(21.2)$ & -1.23 & .224 \\
\hline
\end{tabular}

Note: All age variables in years; SRS: social responsiveness scale; ASD: autism spectrum disorder; HISEI: highest international socioeconomic index. 
agnosed with ADHD (F90.0, 16.5\%), one with ADD (F98.8, 4.2\%), one with hyperkinetic conduct disorder (F90.1, 4.2\%), one with ODD (F91.3, 4.2\%), one with a myofunctional disorder (F82.2, 4.2\%), one with phonophobia (F40.2, 4.2\%), and three with intellectual disability (F70.0, 12.5\%).

Most children had started school (79.2\%); four children were going to kindergarten (16.7\%). $87.5 \%$ of the children lived with at least one sibling in the same household. The mean age at the beginning of the intervention was 9.42 years ( $S D$ 8.6, range $4-19$ years). Five children $(20.8 \%$ ) had started to use psychotropic medication (risperidone and/or methylphenidate) or had a change of dosage during the study. Twenty parents were in a permanent relationship, four lived as single parent (16.7\%). Six parents (25\%) were immigrants and spoke a non-German native language. Three parents (12.5\%) reported having psychiatric problems or psychiatric diagnoses (depression, psychosomatic problems, schizophrenia). Three parents $(12.5 \%)$ had taken part in another unspecific parent training before.

\section{Intervention (FAUT-E)}

The Frankfurt Autism-Specific Parent Training (FAUT-E) is a manualized psychoeducative parent training for parents of children with ASD, especially conceptualized for newly diagnosed children and adolescents (Schlitt et al., 2014). Eight sessions à 120 minutes are delivered by two behaviorally trained therapists over 3 months. The obligatory six basic modules deal with the following topics: (1) ASD symptoms and disorder, (2) positive parent-child interaction, (3) behavior analysis, (4) learning strategies I: structure and orientation, (5) learning strategies II: consequences, and (6) parental empowerment. Nine flexible modules provide specific training on: information on effective intervention (E1), preschool and school support (E2), laws and rights (E3), and role of siblings (E4). For children with ID, additional topics include language training (KK1), and toilet training (KK2). For high-functioning children additional topics are understanding social situations (SK1), information to the child about her/his own diagnosis (SK2), and puberty (SK3).

\section{Outcome Measures}

To record basic demographic information, parents were given a questionnaire before training regarding child characteristics and parental characteristics. Socioeconomic status was calculated by using the (highest) international socioeconomic index ([H]ISEI; Ganzeboom \& Treiman, 1996) based on parental income and profession.

German versions of international standardized questionnaires were used to assess different aspects of child behavior, parental stress, and parental competence.
The Aberrant Behavior Checklist (ABC; Aman \& Singh, 1994) assesses child behavior problems. The items are rated on a 4-point Likert-type scale ranging from 0 (no problem) to 3 (severe problem). Scores were calculated for the total score and five subscales: Irritability, Hyperactivity, Lethargy/Social withdrawal, Stereotypic behavior, and Inappropriate speech.

The Eyberg Child Behavior Inventory (ECBI; Beelmann et al., 2006; Eyberg \& Pincus, 1999) contains descriptions of 36 child problem behaviors. The parent indicates how often each behavior is present on a 7-point Likert-type scale, ranging from 1 (never) to 7 (always). The parent also indicates whether the specific behavior is currently a problem by circling "Yes" or "No" for the respective behavior. The ECBI provides two summary scores: an intensity score and a problem score.

Psychiatric symptoms in different domains were assessed with the Child Behavior Checklist (CBCL 4-18; Arbeitsgruppe Deutsche Child Behavior Checklist, 1998a), an internationally validated and widely used parent report form with 113 items. Responses are recorded on a 3-point Likert-type scale, ranging from 0 (not at all) to 2 (all the time). A total score, second-order scores for internalizing and externalizing problems, and first-order scores for various behavioral and emotional problems (withdrawn, somatic complaints, anxious/ depressed, social problems, thought problems, attention problems, delinquent behavior and aggressive behavior) are computed.

The Home Situation Questionnaire (HSQ/EF-PF; Barkley \& Edelbrock, 1987; Döpfner et al., 2006) assesses variation in problem behavior occurrence and severity across settings. The HSQ contains 16 items describing typical situations at home. Parents are asked to indicate whether problem behaviors occur in these situations and, if so, to rate the severity on a 9-point Likert-type scale, ranging from 1 (mild) to 9 (severe).

The Parenting Scale (PS/EFB; Arnold et al., 1993; Naumann et al., 2010) was developed to identify dysfunctional parenting practices. Parents are supposed to indicate how they would react to problematic child behavior in 35 hypothetical situations. The items are rated on a 7-point Likerttype scale ranging from 1 to 7 . Items are assigned to one of the three scales Laxness, Overreactivity, and Verbosity and are also summarized into a Total score.

The German adaptation of the Problem Setting and Behavior Checklist (PSBC/VER; Sanders et al., 2000) is a 27-item rating scale that assesses how confident parents feel dealing with child behavior problems in various settings on a 5-point Likert-type scale, ranging from 0 to 3.

The Parenting Stress Index (PSI/EBI; Abidin, 1990; Tröster, 2010) assesses child problem behavior and parental limitations of functioning and competences by 48 items 
rated on a 5-point Likert-type scale, ranging from 1 (strongly disagree) to 5 (strongly agree).

\section{Statistical Analyses}

Differences in variables between the study participants and the excluded group were calculated using independent $t$-tests or nonparametric Mann-Whitney $U$-tests, as appropriate. Differences in frequency distributions were described by $\chi^{2}$ tests. Mixed linear models were calculated for all outcome variables of interest to adjust the analysis for the correlation between observations of the same patient at different timepoints and also to consider observations with missing values appropriately. An unstructured correlation structure was assumed. The variable "time" as predictor of interest was included as a fixed effect (categorical variable with 4 categories T0 to T3). In addition, Social Responsiveness Scale (SRS; Constantino et al., 2005) $t$-scores collected at TO; chronological age and IQ were included as covariates (fixed effects). The adjusted mean values and standard errors were derived from these models for all timepoints. The effect size calculation is related to Cohen's $d$, generated by using the standard error and depending sample size. All analyses were carried out for the total sample and - as sensitivity analyses - for the sample excluding participants who were prescribed psychiatric medication. All analyses were carried out using $\mathrm{R}$ (version 3.1.1) in combination with the packages lme 4 (version 1.1-12), lmerTest (version 2.0-32), MASS (version 7.345), xtable (version 1.8-2), and IBM SPSS Statistics 22. Because this study is a pilot study aiming at establishing effect sizes, no adjustment for multiple testing was done.

\section{Results}

Children's and parents' measures over the four timepoints are shown in Table 2 and Table 3.

Results were reported only when the effect of time in the mixed model was significant and additionally post-hoc testing confirmed significant change between $\mathrm{T} 1$ and $\mathrm{T} 2$ or between $\mathrm{T} 1$ and $\mathrm{T} 3$. Changes between $\mathrm{T} 0$ and $\mathrm{T} 2$ or between T0 and T3 were considered only if there was no significant change between $\mathrm{T} 0$ and $\mathrm{T} 1$ (as indicated by posthoc tests). Subsequently, significant changes between T2/ $\mathrm{T} 3$ and $\mathrm{TO}$ were not considered if a significant change had already occurred between $\mathrm{T} 0$ and $\mathrm{T} 1$.

In the parent-rated $\mathrm{ABC}$, the Total score $(\mathrm{ES}=.73, p<$ $.05)$ as well as the subscales Irritability ( $\mathrm{ES}=.54, p<.05)$, Lethargy $(\mathrm{ES}=.42, p<.05)$, and Hyperactivity $(\mathrm{ES}=.64, p$ $<.05)$ decreased between T1 and T2. The subscale Inappropriate speech decreased between $\mathrm{T} 1$ and $\mathrm{T} 3(\mathrm{ES}=.56, p$ $<.05)$. Pre-post effects were maintained at 3 months follow-up for the Total score $(\mathrm{ES}=.51, p<.05)$ and Hyperacti- vity $(\mathrm{ES}=.47, p<.05)$. No or only very small changes between TO and T1 were observed. Similar effects were observed by sensitivity analysis without the data of children receiving medication (data not shown). The decrease in the ABC Total score was influenced by the fixed effect SRS Total score $(\mathrm{ES}=.37, p<.05)$. The decrease in the subscales Irritability and Hyperactivity was not influenced by the fixed effects SRS Total score, age or IQ. The decrease in the subscale Lethargy was influenced by SRS Total score $(\mathrm{ES}=.43, p<.05)$. The decrease in the subscale Inappropriate speech was influenced by age $(\mathrm{ES}=.27, p<.05)$ and IQ $(\mathrm{ES}=.25, p<.05)$.

In the parent-rated $C B C L 4-18$, the Total score $(E S=.70$, $p<.05)$ as well as the subscales Internalizing (ES $=.66, p<$ $.05)$, Withdrawn (ES $=.78, p<.5)$ and Anxious/depressed (ES $=.46, p<.05)$ decreased between T0 and T2. The subscale Withdrawn decreased between $\mathrm{T} 1$ and $\mathrm{T} 2(\mathrm{ES}=$ $.43, p<.05)$. Pre-post effects were maintained for subscales Internalizing and Withdrawn. The decrease in the CBCL Total score was influenced by the fixed effect SRS Total score $(\mathrm{ES}=.32, p<.05)$, the decrease in the subscale Internalizing was influenced by SRS Total score ( $\mathrm{ES}=.35, p$ $<.05)$ and age $(\mathrm{ES}=.28, p<.05)$. The decrease in the subscales Withdrawn and Anxious depressed was also influenced by SRS Total score (Withdrawn: ES $=.47, p<.05$, Anxious depressed: $\mathrm{ES}=.25, p<.05)$ and age (Withdrawn: $\mathrm{ES}=.36, p<.05$, Anxious depressed: $\mathrm{ES}=.25, p<.05)$.

In the ECBI, the Problem score showed a decrease between T1 and T3 (ES = .46, $p<.05)$, with a small, not significant, effect between T1 and T2. Similar effects were observed by sensitivity analysis without the data of children receiving medication (data not shown). SRS Total score, age and IQ had no influence on these results.

No other medium to large effects were observed for the remaining outcome measures.

\section{Parental Outcome Measures}

Compared to T1, no significant change was found for any of the obtained parental measures (see Table 3).

\section{Discussion}

This study provides first evidence that the German parent training program FAUT-E for parents of children with ASD may have medium and persistent effects on the child's challenging behavior, as observed by the parents themselves. Pre-post, the Total score of the ABC and the subscales Irritability, Lethargy, Hyperactivity and Inappropriate speech decreased, and the Total score and the Hyperactivity subscale maintained the improvement with a medium effect size at $\mathrm{T} 3$ compared to $\mathrm{T} 0$ and $\mathrm{T} 1$. SRS Score (fixed effect in mixed model) as a marker for symptom severity had a small 
Table 2. Change of child's behavior.

\begin{tabular}{|c|c|c|c|c|c|c|c|c|c|c|c|c|c|c|c|}
\hline \multirow[b]{3}{*}{ Variables } & \multirow{2}{*}{\multicolumn{3}{|c|}{$\begin{array}{l}\text { Baseline } \\
\text { T1-T0 }\end{array}$}} & \multicolumn{6}{|c|}{ Pre-post } & \multicolumn{6}{|c|}{ Follow-up } \\
\hline & & & & $\mathrm{T} 2-\mathrm{T} 1$ & & & T2-T0 & & & T3-T1 & & & T3-T0 & & \\
\hline & Coef & $p$ & ES & Coef & $p$ & ES & Coef & $p$ & ES & Coef & $p$ & ES & Coef & $p$ & ES \\
\hline \multicolumn{16}{|c|}{ Aberrant behavior checklist (ABC) } \\
\hline Total score & -0.31 & .907 & -.03 & -9.11 & .001 & -.73 & -9.42 & .001 & -.72 & -6.89 & .018 & -.51 & -7.21 & .017 & -.51 \\
\hline Irritability & -0.53 & .602 & -.11 & -2.53 & .012 & -.54 & -3.06 & .004 & -.62 & -0.30 & .782 & -.06 & -0.82 & .459 & -.16 \\
\hline Lethargy & -0.51 & .566 & -.12 & -1.74 & .048 & -.42 & -2.25 & .015 & -.52 & -.041 & .141 & -.31 & -1.92 & .054 & -.41 \\
\hline $\begin{array}{l}\text { Stereotypic } \\
\text { behavior }\end{array}$ & 0.05 & .915 & .02 & -0.31 & .451 & -.16 & -0.27 & .539 & -.13 & -0.55 & .222 & -.26 & -0.51 & .279 & -.23 \\
\hline Hyperactivity & 0.11 & .932 & .02 & -3.94 & .003 & -.64 & -3.83 & .006 & -.59 & -3.38 & .019 & -.50 & -3.26 & .029 & -.47 \\
\hline $\begin{array}{l}\text { Inappropriate } \\
\text { speech }\end{array}$ & 0.48 & .252 & .24 & -0.55 & .172 & -.29 & -0.08 & .858 & -.04 & -1.18 & .009 & -.56 & -0.70 & .125 & -.32 \\
\hline \multicolumn{16}{|c|}{ Child behaviour checklist 4-18 (CBCL) } \\
\hline Total score & -1.76 & .116 & -.33 & -2.02 & .059 & -.40 & -3.78 & .001 & -.70 & -0.29 & .803 & -.05 & -2.05 & .097 & -.35 \\
\hline Internalizing & -2.15 & .096 & -.35 & -1.93 & .118 & -.33 & -4.08 & .002 & -.66 & -0.99 & .459 & -.16 & -3.14 & .028 & -.47 \\
\hline Externalizing & -0.87 & .467 & -.15 & -0.43 & .704 & -.80 & -1.30 & .286 & -.23 & 1.36 & .275 & .23 & 0.49 & .707 & .08 \\
\hline Withdrawn & -2.90 & .074 & -.38 & -3.12 & .045 & -.43 & -6.01 & .000 & -.78 & -0.65 & .695 & -.08 & -3.55 & .045 & -.43 \\
\hline $\begin{array}{l}\text { Somatic } \\
\text { complaints }\end{array}$ & -0.96 & .522 & -.13 & -0.85 & .551 & -.13 & -1.81 & .235 & -.25 & -2.19 & .164 & -.29 & -3.15 & .059 & -.40 \\
\hline $\begin{array}{l}\text { Anxious/ } \\
\text { depressed }\end{array}$ & -2.05 & .101 & -.35 & -0.72 & .543 & -.13 & -2.76 & .031 & -.46 & -0.32 & .806 & -.05 & -2.36 & .085 & -.37 \\
\hline $\begin{array}{l}\text { Social } \\
\text { symptoms }\end{array}$ & -1.97 & .213 & -.26 & -0.81 & .589 & -.11 & -2.78 & .085 & -.37 & 0.91 & .579 & .12 & -1.06 & .539 & -.13 \\
\hline $\begin{array}{l}\text { Thought } \\
\text { problems }\end{array}$ & 1.25 & .457 & .16 & -0.23 & .283 & -.23 & -0.47 & .783 & -.06 & 0.07 & .967 & .01 & 1.32 & .474 & .15 \\
\hline $\begin{array}{l}\text { Delinquent } \\
\text { behavior }\end{array}$ & 0.19 & .860 & .04 & -0.36 & .728 & -.07 & -0.17 & .879 & -.03 & 0.35 & .758 & .07 & 0.54 & .651 & -.09 \\
\hline $\begin{array}{l}\text { Aggressive } \\
\text { behavior }\end{array}$ & -0.41 & .752 & -.07 & -1.11 & .366 & -.19 & -1.52 & .249 & -.24 & 0.45 & .740 & .07 & 0.04 & .979 & .01 \\
\hline
\end{tabular}

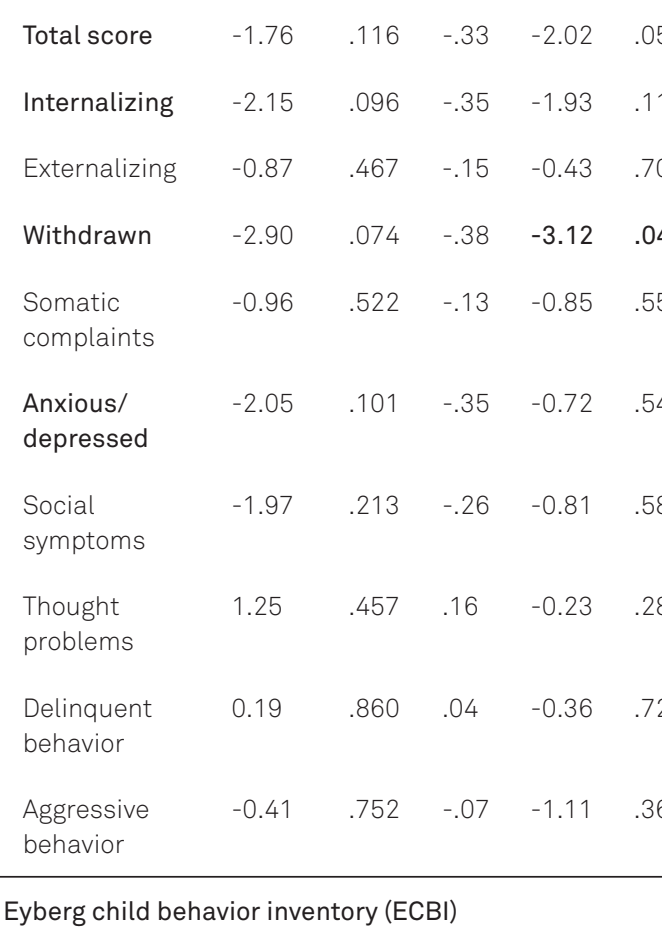

$\begin{array}{lllllllllllllll}-1.93 & .666 & -.09 & -4.04 & .344 & -.20 & -5.98 & .184 & -.28 & -2.96 & .540 & -.13 & -4.89 & .326 & -.21\end{array}$

$\begin{array}{llllllllllllllllll}\text { Problem } & -0.24 & .824 & -.05 & -1.57 & .138 & -.31 & -1.81 & .103 & -.35 & -2.60 & .031 & -.46 & -2.85 & .022 & -.49\end{array}$

Home Situation Questionnaire (HSQ)

\begin{tabular}{|c|c|c|c|c|c|c|c|c|c|c|c|c|c|c|c|}
\hline $\begin{array}{l}\text { Problem } \\
\text { situations }\end{array}$ & -1.69 & .058 & -.40 & 0.52 & .528 & .13 & -1.17 & .187 & -.28 & 0.58 & .531 & .13 & -1.11 & .259 & -.24 \\
\hline Intensity & -12.81 & .001 & -.72 & -2.61 & .455 & -.16 & -15.42 & .000 & -.87 & 1.56 & .694 & .08 & -11.25 & .008 & -.57 \\
\hline
\end{tabular}

Note: Adjusted means and standard errors are shown. Significant pre-post changes compared to T1 (and T0) are highlighted in bold. Significant changes between T2/T3 and T0 were not considered if a significant change had already occurred between T0 and T1. T0 = 2 months before training; T1 = before start of the training; T2 = directly after end of training; T3 = 2-3 months after end of the training. ES = Effect size, Cohen's $d$. 
Table 3. Change in parental outcome measures

\begin{tabular}{|c|c|c|c|c|c|c|c|c|c|c|c|c|c|c|c|c|}
\hline \multirow[b]{3}{*}{ Variables } & & \multicolumn{3}{|c|}{ Baseline } & \multicolumn{6}{|c|}{ Pre-post } & \multicolumn{6}{|c|}{ Follow-up } \\
\hline & & \multicolumn{3}{|l|}{ T1-T0 } & \multicolumn{3}{|l|}{$\mathrm{T} 2-\mathrm{T} 1$} & \multicolumn{3}{|l|}{ T2-T0 } & \multicolumn{3}{|l|}{ T3-T1 } & \multicolumn{3}{|l|}{ T3-T0 } \\
\hline & & Coef & $p$ & ES & Coef & $p$ & ES & Coef & $p$ & ES & Coef & $p$ & ES & Coef & $p$ & ES \\
\hline Parenting scale & & -0.37 & .754 & -.07 & -0.91 & .414 & -.17 & -1.28 & .281 & -.23 & -1.65 & .191 & -.27 & -2.03 & .126 & -.32 \\
\hline $\begin{array}{l}\text { Problem Setting a } \\
\text { Checklist }\end{array}$ & havior & 6.08 & .185 & .27 & 0.13 & .976 & .01 & 6.21 & .176 & .29 & 7.18 & .140 & .31 & 13.26 & .010 & .55 \\
\hline \multirow{3}{*}{$\begin{array}{l}\text { Parenting Stress } \\
\text { Index }\end{array}$} & Total & 2.48 & .467 & .15 & -0.48 & .881 & -.03 & 0.61 & .809 & .05 & 0.18 & .961 & .01 & 1.75 & .531 & .13 \\
\hline & Parent & 2.82 & .217 & .26 & -1.43 & .502 & -.14 & 1.39 & .542 & .13 & -1.74 & .471 & -.15 & 1.08 & .668 & .09 \\
\hline & Child & 1.26 & .616 & .11 & -0.65 & .782 & -.06 & 1.99 & .557 & .12 & 0.48 & .855 & .04 & 2.65 & .481 & .15 \\
\hline
\end{tabular}

Note: Adjusted means and standard errors are shown. T0 = 2 months before training; $\mathrm{T} 1$ = before start of the training; $\mathrm{T} 2$ = directly after end of training; $\mathrm{T} 3$ = 2-3 months after end of the training. ES = Effect size, Cohen's $d$.

effect on most of the significantly decreased scores, leading to the conclusion that the success of the training is not independent of symptom severity in all areas. IQ and age had small effects on a few subscales.

For the ECBI Problem score, a medium change was only found at follow-up (T3) compared to T0 and T1. No persisting change in other child's behavior symptoms or regarding parent related measures was observed.

The parent-reported decrease of the child's challenging behavior subscale Hyperactivity measured at follow-up was similar to findings of the parent training described by Bearss et al. (2015) $(E S=0.64$ vs ES $=0.43)$. Other authors also reported significant reduction of child's challenging behavior - measured by the developmental behavior checklist (DBC; Einfeld \& Tonge, 1995) - directly after intervention (Pillay et al., 2011).

The reduced ECBI Problem score in this study between T1 and follow-up could indicate that the parents needed some time after the training to implement more effective parenting strategies, and that, at the same time, those strategies need to be practiced to become effective. In contrast to studies on Stepping Stones Triple P program for autism, the ECBI Intensity scale did not decrease after FAUT-E (Sprenger et al., 2015; Whittingham et al., 2009). The studies cited showed improvement of both subscales directly after treatment. Compared to our study, the Triple P studies showed higher baseline values, which could partially account for more improvement than was possible in our study.

Parental stress was stable over the course of the training, which indicates that the program did not increase parental burden beyond the diagnosis of the child. Other more intensive and time-demanding parental programs led to increased parental stress during training (Rivard et al., 2016). Contrary to HPP, C-Hope, Stepping Stones Trip- le P program for autism, and the parent training described by Iadarola et al. (2018), a reduction of parental stress was not found for FAUT-E (Iadarola et al., 2018; Kuravackel et al., 2018; Leung et al., 2016; Schrott et al., 2019). Studies that reported reduced parental stress level offered additional individual sessions (Iadarola et al., 2018; Schrott et al., 2019; Sprenger et al., 2015; Tonge et al., 2006). A possible delayed effect was noticed in the PSBC/VER. Here, the baseline to follow-up development showed a significant increase, indicating a possible time delay of new parenting methods showing measurable results in children's behaviors. More confidence in dealing with child behavior problems likely occurs later because parents need time to test recommended strategies at home and find their own way regarding their individual situation. Similar results regarding parental efficacy at follow-up were reported in other studies (Tonge et al., 2006; Whittingham et al., 2009).

Most psychoeducative parent trainings are teaching similar evidence-based contents to the parents, such Stepping Stones Triple P for autism (Schrott et al., 2019; Whittingham et al., 2009). Additionally, FAUT-E focuses on the empowerment of the parents as well as on acceptance and commitment strategies. Besides providing information about ASD and useful strategies for behavior management, positive parent-child interaction and playtime is enhanced (in session 2), and parents are encouraged to establish time for themselves to set free new own resources (in session 6).

\section{Limitations}

The strongest limitations of the present study are the small and heterogenous sample, the missing control group, and the unblinded outcome measures. In addition, all outcome 
measures showed a high variability, which is common in intervention studies on children with ASD. Results should thus be interpreted with caution. Similar to our study, however, most studies on parent group trainings used parent reports as outcome measures. Reasons for this approach could be lower costs when using parents reports instead of direct observation measures and a lack of direct observation outcome measures with adequate sensitivity to behavioral change.

Several children received a change in medication during the study. Because the medication is also targeting especially challenging and hyperactive behavior, it cannot be fully excluded that the observed change in the parent-rated $\mathrm{ABC}$ and ECBI are due to medication, not parent training effects (Aman et al., 2009). Still, sensitivity analyses showed comparable effect sizes in the subgroup without medication, which supports real effects of the parent training on the child's behavior.

From the present study, $35 \%$ of the parents aiming at participating in the training had to be excluded from analysis because of missing questionnaire data or because they missed three consecutive parent training sessions in a row. These parents had younger children with ASD; otherwise, no statistically significant differences were observed. This highlights the importance of evaluating factors which might hinder parents to participate in a parent training.

FAUT-E was delivered by the authors KB and SS, who also developed the training together with CMF. Further studies should be done by independent groups and should include larger samples and a randomized control group. Information on parent's expectations, feasibility of implementation with low-resourced families, families with young children and in different clinical settings, as well as treatment fidelity measures should also be obtained.

\section{Conclusion}

Because FAUT-E is a newly developed intervention, the aim of the study was to establish pre-post effect sizes and to compare these to other psychoeducational parent trainings that are not available in German. An uncontrolled repeated measurement design was implemented to differentiate between effects of waiting for the intervention and of the intervention itself. No explicit feasibility study was done, which would aim more extensively at patient/parent and therapist acceptability, fidelity of therapists to manual, and collecting data on how many parents would be willing to attend a parent training. Taken together, medium effect sizes regarding reduction of the child's challenging behavior were reported by parents. In light of internation- al, comparable group-based psychoeducative behavioral interventions for parents of children with ASD, it can be concluded that psychoeducative behavioral parent trainings should be implemented in the everyday care of children with ASD and their parents, especially in the presence of challenging behavior problems. The group format as well as the low number of sessions allows implementing FAUT-E in different clinical settings. The additional flexible modules make the program suitable for all parents of children with ASD, independent of age, language, or cognitive level. The flexible modules can be used to adapt the program according to symptom severity. Compared to other programs, this is another advantage of FAUT-E, because some of the other programs are conceptualized or evaluated only for children of a specific age range, i.e. , preschool-aged children (Feinberg et al., 2014; Leung et al., 2016; Rivard et al., 2016; Sprenger et al., 2015; Tonge et al., 2014) or for children with a small range of symptom variability, i.e. verbal and high functioning (Whittingham et al., 2009).

\section{References}

Abidin, R.R. (1990). Parenting stress index-short form. Pediatric Psychology Press.

Arbeitsgruppe Deutsche Child Behavior Checklist. (1998a). Elternfragebogen über das Verhalten von Kindern und Jugendlichen. Deutsche Bearbeitung der Child Behavior Checklist (CBCL/418). 2. Auflage mit deutschen Normen, bearbeitet von M. Döpfner, J. Plück, S. Bölte, K. Lenz, P. Melchers \&. K. Heim. Köln: Arbeitsgruppe Kinder-, Jugend- und Familiendiagnostik (KJFD).

Aman, M.G. \& Singh, N.N. (1994). Aberrant behavior checklist community. Supplementary manual. Slosson Educational Publications.

Aman, M.G., Mcdougle, C.J., Scahill, L., Handen, B., Arnold, L.E., Johnson, C. et al. [please supply all authors] (2009). Medication and parent training in children with pervasive developmental disorders and serious behavior problems: Results from a randomized clinical trial. Journal of the American Academy of Child \& Adolescent Psychiatry, 48, 1143-1154.

American Psychiatric Association (APA). (2013). Diagnostic and statistical manual of mental disorders: DSM-5 (5th ed). Author.

Arnold, D. S., O'Leary, S. G., Wolff, L. S. \& Acker, M. M. (1993). The Parenting Scale: A measure of dysfunctional parenting in discipline situations. Psychological Assessment, 5, 137-144.

Barkley, R.A. \& Edelbrock, C.S. (1987). Assessing situational variation in children's behavior problems: The Home and School Situations Questionnaires. Advances in Behavioral Assessment of Children and Families, 3, 157-176.

Bearss, K., Burrell, T.L., Stewart, L., \& Scahill, L. (2015). Parent training in autism spectrum disorder: What's in a name? Clinical Child and Family Psychology Review, 18, 170-182.

Beelmann, A., Lösel, F., Stemmler, M. \& Jaursch, S. (2006). Beurteilung von sozialen Verhaltensproblemen und Erziehungsschwierigkeiten im Vorschulalter. Diagnostica, 52, 189-198.

Bölte, S., Rühl, D., Schmötzer, G., \& Poustka, F. (2006). Diagnostisches Interview für Autismus - revidiert: ADI-R; deutsche Fas- 
sung des Autism Diagnostic Interview - Revised (ADI-R) von Michael Rutter, Ann Le Couteur und Catherine Lord; Manual. Huber/Hogrefe.

Bölte, S. \& Poustka, F. (2006). Fragebogen zur sozialen Kommunikation - Autismus Screening (FSK). Deutsche Fassung des Social Communication Questionnaire (SCQ). Huber.

Constantino, J.N., \& Gruber, C.P. (2005). Social Responsiveness Scale (SRS). Western Psychological Services.

Döpfner, M., Lehmkuhl, G. \& Steinhausen, H.-C. (2006). Kinder-Diagnostik-System (KIDS). Aufmerksamkeitsdefizit - und Hyperaktivitätsstörung (ADHS). Hogrefe.

Einfeld, S.L., \& Tonge, B.J. (1995). The Developmental Behavior Checklist: The development and validation of an instrument to assess behavioral and emotional disturbance in children and adolescents with mental retardation. Journal of Autism and Developmental Disorders, 25, 81-104.

Eyberg, S. M. \& Pincus, D. (1999). Eyberg Child Behavior Inventory and Sutter-Eyberg Student Behavior Inventory-Revised: Professional manual. Psychological Assessment Resources.

Fairthorne, J., Klerk, N. de \& Leonard, H. (2015). Health of mothers of children with intellectual disability or autism spectrum disorder: A review of the literature. Medical Research Archives (3). Advance online publication. https://doi.org/10.18103/mra. v0i3.204

Feinberg, E., Augustyn, M., Fitzgerald, E., Sandler, J., Ferreira-Cesar Suarez, Z., Chen, N. et al. [please supply all authors] (2014). Improving maternal mental health after a child's diagnosis of autism spectrum disorder. JAMA Pediatrics, 168, 40[only 1 page long?].

Ganzeboom, H.B. \& Treiman, D.J. (1996). Internationally comparable measures of occupational status for the 1988 International Standard Classification of Occupations. Social Science Research, 25, 201-239.

ladarola, S., Levato, L., Harrison, B., Smith, T., Lecavalier, L., Johnson, C., et al. [please supply all authors] (2018). Teaching parents behavioral strategies for autism spectrum disorder (ASD): Effects on stress, strain, and competence. Journal of Autism and Developmental Disorders, 48, 1031-1040.

Kendall, T., Megnin-Viggars, O., Gould, N., Taylor, C., Burt, L.R. \& Baird, G. (2013). Management of autism in children and young people: summary of NICE and SCIE guidance. BMJ, 347, f4865.

Kuravackel, G.M., Ruble, L.A., Reese, R.J., Ables, A.P., Rodgers, A.D., \& Toland, M.D. (2018). Compass for hope: Evaluating the effectiveness of a parent training and support program for children with ASD. Journal of Autism and Developmental Disorders, 48, 404-416.

Leung, C., Chan, S., Lam, T., Yau, S. \& Tsang, S. (2016). The effect of parent education program for preschool children with developmental disabilities: A randomized controlled trial. Research in Developmental Disabilities, 56, 18-28.

Lord, C., Rutter, M., DiLavore, P.C. \& Risi, S. (1999). ADOS. Autism Diagnostic Observation Schedule. Manual. Western Psychological Services.

Lord, C., Rutter, M. \& Le Couteur, A. (1994). Autism Diagnostic Interview-Revised: A revised version of a diagnostic interview for caregivers of individuals with possible pervasive developmental disorders. Journal of Autism and Developmental Disorders, 24, 659-685.

McMillin, S. E., Bultas, M.W., Wilmott, J., Grafeman, S. \& Zand, D.h. (2015). Rapid-response parenting intervention in diagnostic centers as a patient-centered innovation for autism spectrum disorders. Journal of Autism and Developmental Disorders, 45, 269-271.

Naumann, S., Bertram, H., Kuschel, A., Heinrichs, N., Hahlweg, K. \& Döpfner, M. (2010). Der Erziehungsfragebogen (EFB). Diagnostica, 56, 144-157.
Pillay, M., Alderson-Day, B., Wright, B., Williams, C. \& Urwin, B. (2011) Autism Spectrum Conditions - Enhancing Nurture and Development (ASCEND): An evaluation of intervention support groups for parents. Clinical Child Psychology and Psychiatry, 16, 5-20.

Rivard, M., Morin, M., Mercier, C., Terroux, A., Mello, C. \& Lépine, A. (2016). Social validity of a training and coaching program for parents of children with autism spectrum disorder on a waiting list for early behavioral intervention. Journal of Child and Family Studies, 26, 877-887.

Rühl, D. (2004). ADOS: Diagnostische Beobachtungsskala für autistische Störungen. Manual. Deutsche Fassung der Autism Diagnostic Observation Schedule. Huber.

Sanders, M.R., Markie-Dadds, C., Tully, L.A. \& Bor, W. (2000). The Triple P-Positive Parenting Program: A comparison of enhanced, standard, and self-directed behavioral family intervention for parents of children with early onset conduct problems. Journal of Consulting and Clinical Psychology, 68, 624-640.

Schlitt, S., Berndt, K. \& Freitag, C. M. (2014). Das Frankfurter Autismus-Elterntraining (FAUT-E): Psychoedukation, Beratung und therapeutische Unterstützung (Kohlhammer Manuale). Kohlhammer.

Schrott, B., Kasperzack, D., Weber, L., Becker, K., Burghardt, R. \& Kamp-Becker, I. (2019). Effectiveness of the Stepping Stones Triple P Group Parenting Program as an additional intervention in the treatment of autism spectrum disorders: Effects on parenting variables. Journal of Autism and Developmental Disorders, 49, 913-923.

Simonoff, E., Jones, C.R., Pickles, A., Happé, F., Baird, G. \& Charman, T. (2012). Severe mood problems in adolescents with autism spectrum disorder. Journal of Child Psychology and Psychiatry, and Allied Disciplines, 53,1157-1166.

Sprenger, L., Becker, K., Heinzel-Gutenbrunner, M., Mingebach, T. Otterbach, S., Peters, M. et al. (2015). Ist das "Stepping-Stones/ Triple P“-Elterntraining eine sinnvolle, ergänzende Intervention in der Behandlung von Kindern mit Autismus-Spektrum-Störungen? Kindheit und Entwicklung, 24, 28-36.

Stuttard, L., Beresford, B., Clarke, S., Beecham, J. \& Morris, A. (2016). An evaluation of the Cygnet parenting support programme for parents of children with autism spectrum conditions. Research in Autism Spectrum Disorders, 23, 166-178.

Tonge, B., Brereton, A., Kiomall, M., Mackinnon, A., King, N. \& Rinehart, N. (2006). Effects on parental mental health of an education and skills training program for parents of young children with autism: A randomized controlled trial. Journal of the American Academy of Child \& Adolescent Psychiatry, 45, 561-569.

Tonge, B., Brereton, A., Kiomall, M., Mackinnon, A. \& Rinehart, N.J. (2014). A randomised group comparison controlled trial of "preschoolers with autism": A parent education and skills training intervention for young children with autistic disorder. Autism, 18, 166-177.

Tröster, H. (2010). Eltern-Belastungs-Inventar (EBI). Deutsche Version des Parenting Stress Index (PSI) von R. R. Abidin. Hogrefe.

Whittingham, K., Sofronoff, K., Sheffield, J. \& Sanders, M. R. (2009). Stepping Stones Triple P: An RCT of a parenting program with parents of a child diagnosed with an autism spectrum disorder. Journal of Abnormal Child Psychology, 37, 469-480.

\section{History}

Manuscript submitted: 31.01 .2020

Accepted after revision: 24.11.2020

Published online: 11.01.2021

\section{Funding}

All funding was Internal with no external funding source. 


\section{Ethical approval}

All procedures performed in studies involving human participants were in accordance with the ethical standards of the institutional and/or national research committee and with the 1964 Helsinki Declaration and its later amendments or comparable ethical standards. This article does not contain any studies with animals performed by any of the authors.

\section{Informed consent}

Informed consent was obtained from all parents included in the study.

\section{Conflicts of interest}

The authors $\mathrm{KJ}$ and LU do not report any conflict of interest. The authors SS, KB, and CMF developed the FAUT-E approach and receive royalties for the intervention manual. CMF has served as consultant for Desitin and Roche and receives royalties for books on ASD, ADHD, and MDD. JM and JK receive royalties for a book on ASD. CS receives royalties for a manual on a parent training for parents of children with psychiatric disorders.

\section{Dr. med. Juliane E. Medda}

Deutschordenstr. 50

60528 Frankfurt am Main

Germany

juliane.medda@posteo.de 